\title{
Human Stress and StO2: Database, Features, and Classification of Emotional and Physical Stress
}

\author{
Xinyu Liu ${ }^{1,2}$, Yuhao Shan ${ }^{1,2}$, Min Peng ${ }^{1,2}$, Huanyu Chen ${ }^{1,2}$ and Tong Chen $1,2,3,4, *$ (I) \\ 1 Chongqing Key Laboratory of Non-Linear Circuit and Intelligent Information Processing, \\ Southwest University, Chongqing 400715, China; liuxinyu1223@email.swu.edu.cn (X.L.); \\ fgyuhao@email.swu.edu.cn (Y.S.); peng2014m@email.swu.edu.cn (M.P.); \\ chy199315@email.swu.edu.cn (H.C.) \\ 2 School of Electronic and Information Engineering, Southwest University, Chongqing 400715, China \\ 3 Key Laboratory of Cognition and Personality (SWU), Ministry of Education, Chongqing 400715, China \\ 4 Institute of Psychology, China Academy of Sciences, Beijing 100101, China \\ * Correspondence: c_tong@swu.edu.cn
}

Received: 4 July 2020; Accepted: 28 August 2020; Published: 31 August 2020

\begin{abstract}
Emotional and physical stress can cause various health problems. In this paper, we used tissue blood oxygen saturation (StO2), a newly proposed physiological signal, to classify the human stress. We firstly constructed a public StO2 database including 42 volunteers subjected to two types of stress. During the physical stress experiment, we observed that the facial StO2 right after the stress can be either increased or decreased comparing to the baseline. We investigated the StO2 feature combinations for the classification and found that the average StO2 values from left cheek, chin, and the middle of the eyebrow can provide the highest classification rate of $95.56 \%$. Comparison with other stress classification method shows that $\mathrm{StO} 2$ based method can provide best classification performance with lowest feature dimension. These results suggest that facial StO2 can be used as a promising features to identify stress states, including emotional and physical stress.
\end{abstract}

Keywords: human stress database; hyperspectral imaging; tissue oxygen saturation; human stress classification; emotional stress; physical stress

\section{Introduction}

Stress, which refers to a state of tension when individuals are mentally or physically threatened, is a kind of imbalanced homeostasis state. Stress can be divided into psychological stress (also known as emotional stress) and physical stress according to different sources of stressors. Emotional stress comes from internal stimuli, such as the nervousness brought by stage speeches and important exams. Being under emotional stress for a long time is a chronic health challenge to the body. Serious emotional stress can cause some mental illnesses, such as depression. Physical stress comes from external stimuli, such as excessive exercise, working or driving long hours [1]. Short-term physical stress gives people a feeling of exhaustion and listlessness. Being under long-term physical stress will seriously affect our health. Epidemiological studies have pointed out that stress stimulus has a certain relationship with the increased incidence of hypertension, ulcers, accidents, cardiovascular events [2,3], myocardial infarction, diabetes, immunological problems and cancer [4-7].

Since both types of stress have an impact on health, the research of stress detection has attracted the attention of both engineers and psychologists. Depending on the source of signal, human stress detection can be based on external visual appearance as well as internal physiological signals. External appearance features can be extracted from facial expressions, gesture, voice, etc. Internal physiological features can be extracted from common physiological signals, such as ECG, EMG, EEG, 
breath, respiratory, etc. Although the stress detection based on external appearance is intuitive, it is also easily to be disguised, resulting in the failure of detection. Nevertheless, the internal physiological signals are involuntary and hard to manipulate, and usually correspond to true feelings. Therefore, it is more accurate to use physiological signals for stress detection.

In traditional studies, the stress detection based on physiological signals mostly uses contact methods [1,8-11]. In the process of this detection, participants need to wear sensors, which leads to inconvenience in operation and difficulty in promotion and application. In order to improve the convenience, stress detection based on imaging technology has been developing rapidly in recent years. Using some specific imaging technologies to extract physiological signals to detect stress has also achieved good results. Pavlidis et al. [12,13] used thermal imaging (TI) to measure blood flow under skin surface and perspiration of nose to detect stress. McDuff et al. [14] used broadband imaging to measure heart rate variability (HRV) to detect stress. We [15] used hyperspectral imaging (HSI) technology to extract tissue blood oxygen saturation (StO2) to detect psychological stress. Later on Hong et al. [16] used multispectral imaging (MSI) technology to measure the physical stress.

$\mathrm{StO} 2$, one of the latest physiological indicators, is associated closely with stress. When an individual feels stressed, the hypothalamus-pituitary-adrenal (HPA) secretes adrenaline, preparing the body for fight-or-flight. When in a state of stress, heart and lung activity increases, and nutrients such as glucose and oxygen are released in large quantities for muscle activity. At the same time, the blood of the whole body will also have corresponding changes. For example, blood pressure increases, blood thickens, and blood flow speeds up to $300 \sim 400 \%$ [15] for aroused brain, heart, and muscles. The response to stressors will bring about a series of changes in physiological indicators, including facial StO2, which is the basis of this study [15].

Most of the stress detection studies mentioned above focus on one type of stress recognition. However, physical stress and emotional stress share some similar characteristics, such as increased heart rate [15] and breathing rates, muscle tension [17], perspiration [12], which makes it somewhat difficult to distinguish between physical and emotional stress. Meanwhile, relief methods for the two types of stress are completely different [18]. Emotional stress requires proper psychological counseling, while physical stress requires adequate rest and relaxation. It is easy to confuse the two types of stress when the content of stimuli is unknown, or when the selection of physiological features is not appropriate. It is necessary to accurately distinguish between physical stress and emotional stress, and to take the problem-oriented strategy for different types of stress.

One of key factors for distinguishing these two types of stress would be to understand the characteristics of these two, i.e., to find out the distinguishing features among these two. We have investigated $[19,20]$ one of traditional physiological signals, the respiratory signal, to find out some useful features for distinguishing these two types of stress. However, as regard to the latest physiological signal, the $\mathrm{StO} 2$, little work has been done to find out their distinguishing features. In a previous study [15], we found that when the participants were under psychological stress, the average $\mathrm{StO} 2$ values of their forehead were significantly higher than those of the baseline condition. In another pilot study [21], we found that the change trend of $\mathrm{StO} 2$ was different among different participants under physical stress. Some persons have higher $\mathrm{StO} 2$ right after the physical stress than that of baseline, while some have lower ones.

In this paper, we would like to examine in depth the distinguishing $\mathrm{StO} 2$ features of these two and differentiate the two types of stress only by using $\mathrm{StO} 2$ features. To achieve this, we firstly build an $\mathrm{StO} 2$ database including 42 participants' facial $\mathrm{StO} 2$ data, under emotional stress, physical stress, and baseline states. This database is the first $\mathrm{StO} 2$ database including two types of stress and will be publicly available to the research community. We then carefully examine the facial regional StO2 when participants are in stress. The changes of $\mathrm{StO} 2$ due to stress vary from person to person and from face region to region. After that the best combination of regional StO2 were determined by using exhaustive search. By using the group of regional StO2, the emotional stress and physical stress are differentiated. The average accuracy of classifying the two types of stress reaches $95.56 \%$. 
To sum up, the main contributions of this paper have three aspects. Firstly, we provide the first public stress database of StO2 through non-contact HSI, which contains emotional stress and physical stress. Secondly, we found that the changes of facial StO2 vary in different face regions and different persons, and the type of stress also make difference. Thirdly, we tested all the combinations of the seven regional $\mathrm{StO} 2$ features to find the optimal $\mathrm{StO} 2$ feature combination, and classified the two types of stress by only using $\mathrm{StO} 2$ features.

The structure of the rest paper is arranged as follows: Section 2 shows the experimental design and the establishment of $\mathrm{StO} 2$ stress database. Section 3 explores different facial $\mathrm{StO} 2$ changes under different types of stress. Section 4 presents the detection of emotional stress and physical stress, including the detection results and the study of certain feature regions. Conclusion and discussion are given in Section 5.

\section{StO2 Stress Database}

\subsection{Generating Facial StO2 by HSI}

HSI is a technique that performs detailed segmentation on spectral dimensions and enables imaging in hundreds of adjacent narrow bands with a bandwidth of about $10 \mathrm{~nm}$. What is obtained by HSI is a 3D image cube [22-24], which contains not only spatial (two-dimensional) but also spectral (one-dimensional) information. The traditional imaging technique is to image on three broad-band wavelength channels $(\mathrm{R}, \mathrm{G}, \mathrm{B})$, and tends to reduce the color discrimination ability. However, HSI can be performed in hundreds of narrow wavebands, with a strong ability to distinguish colors, coupled with the texture information of the object, making it has a strong ability to distinguish materials [25]. It is based on this characteristic of HSI that we use it to sense and distinguish blood chromophores inside body tissues [26].

When air is inhaled into the lungs, oxygen diffuses in the lung to the blood and binds to deoxygenated hemoglobin to form oxyhemoglobin $\left(\mathrm{HbO}_{2}\right)$ in an unstable and reversible way. When the oxygen detached to diffuse in the cell, the $\mathrm{HbO}_{2}$ complex becomes into deoxygenated hemoglobin $(\mathrm{Hb}) . \mathrm{Hb}$ returns to the heart through the veins and then enters the lungs to bind to the inhaled oxygen again. The body's entire blood will carry out such circulation process. Each hemoglobin molecule can bind to up to four oxygen molecules. Hemoglobin oxygen saturation (SO2) is defined as the ratio of the amount of $\mathrm{HbO}_{2}$ to the total amount of hemoglobin:

$$
\mathrm{SO} 2=\frac{\mathrm{HbO}_{2}}{\mathrm{Hb}+\mathrm{HbO}_{2}}
$$

The $\mathrm{StO} 2$ used in this paper is the $\mathrm{SO} 2$ of microcirculation in tissues, ranging from approximately $60 \%$ of venous $\mathrm{SO} 2$ to $98 \%$ of arterial $\mathrm{SO} 2[15,27,28]$. When the individual performs strenuous exercise or experiences extreme emotions such as fear, the value of $\mathrm{StO} 2$ will deviate from the normal level. For example, the facial StO2 during exercise could be less than $60 \%$. Sufficient work has been done to extract StO2 data by HSI based on Beer-Lambert Law [29-31]. The facial StO2 in this paper is deduced from HSI date by using Modified Beer Lambert Three-Chromosphere model [15,24].

\subsection{Experimental Setup}

The HSI system utilized in this study consists of a Specim VNIR spectrograph (SPECIM, SPECTRAL IMAGING LTD., Oulu, Finland) together with a PixelFly camera (PCO, PCO AG, Kelheim, Gemany) and a home-designed mirror scanning system. The slit of the spectrograph is $30 \mu \mathrm{m}$ width giving a maximum spectral resolution of $2.8 \mathrm{~nm}$. The limit of the spectral sensitivity of the PCO camera ranges from $400 \mathrm{~nm}$ to $1000 \mathrm{~nm}$ with a maximum quantum efficiency yield of $65 \%$ at $650 \mathrm{~nm}$. The size of whole HSI system is about $40 \mathrm{~cm} \times 20 \mathrm{~cm} \times 12 \mathrm{~cm}$. The HSI system images at a fixed number of wavelengths, i.e., from $380 \mathrm{~nm}$ to $800 \mathrm{~nm}$ with a step of $2 \mathrm{~nm}$ (300 wavelengths). It needs $20 \mathrm{~s}$ for the system to record one image cube. 
During the whole imaging process, halogen lights were used as the sole illumination source. The HSI system is about $1 \mathrm{~m}$ away from the participants. The HSI data were obtained when the participants were in still pose. During the imaging, the participants were required to rest their jaws on a jaw bracket just like the ones used in optometry to keep the face still. Twenty seconds are required for the system to record one image cube. Any slight movement of the participants will cause distortion of the $\mathrm{StO} 2$ image, however, this distortion will be adjusted by using a registration operation.

\subsection{Experimental Procedures and Protocols}

Forty-two volunteers participated in the experiment. They were students aged from 18 to 25 at Southwest University, half of whom were male and half female. All participants were healthy, with no color recognition disorder and free from mental and physical illnesses. Prior to the experiments, everyone signed an informed consent. The experimental protocols were approved by the local Ethics Committee of Southwest University. All the experiments were conducted indoors with the room temperature being 26 degrees Celsius (air conditioning was applied), and the data were collected while the participant was sitting in a chair. The participant was given $10 \mathrm{~min}$ to adapt after entering the experimental environment. During the $10 \mathrm{~min}$, the participant was introduced the procedure details of the experiment (see Figure 1). The experiment consisted of three main sessions: the baseline session, the emotional stress session and the physical stress session. A modified Trier Social Stress Test (TSST) was used to stimulate participants' emotional stress. The TSST is a widely used social psychological stress test in the world [32]. Its psychological stress test includes public speaking and numerical calculation. Since there is no professional audience in our experiment, we used a Stroop Color-Word test [33] to replace the public speech. In this paper, timed mental arithmetic and Stroop Color-Word tests [33] were employed as emotional stressors, and each stimulus was randomly presented. Studies have shown that Stroop Color-Word test can effectively stimulate participants' psychological stress $[34,35]$. The Stroop Color-Word test slide shows a color-meaning word (Red, blue, green or yellow) in colors that differ from the meaning of the word, such as the word "Green" in Blue. Participants were asked to randomly choose the meaning or color of the word. Before the test, the participant was told that if he/she made more correct choices than the average, he/she would get CNY20 as a reward. The answer time per slide was $5 \mathrm{~s}$ at the beginning, and gradually decreased to $3.5 \mathrm{~s}$ at the end of the test to increase the difficulty. HSI data of emotional stress was collected as ES. In the physical stress session, participants were asked to perform 30 to 60 self-weight squats as many as they could. After the squats, they returned to their seats immediately and we obtained the facial HSI data of physical stress as PS1. Physical stress data PS2 was taken after the participant had rested for two minutes after the squats. Before each session, there was a baseline session, during which participants were given a five-minute break to adjust their states back to normal. Then HSI data of baseline was collected. After all sessions were completed, participants were asked to evaluate the levels of emotional and physical stress on a scale of 1 to 10. A self-reported score greater than 6 was considered as valid data for subsequent analysis and identification. In our experiments, all participants reported stress levels greater than 6. HSI data were collected at the end of each experimental session. The baseline data corresponding to each session were collected to remove individual differences.



Figure 1. Experimental procedure. 
The emotional stress test was performed first and then the physical stress was performed (See Figure 1). The sequence of these two tests was not randomized. To eliminate the any possible effect of emotional stress test on the physical stress, there are five minutes for the participant to prepare the physical stress and another five minutes to break so as to restore himself/herself back to normal. That is to say from the end of the emotional stress test to the beginning of physical stress, there are ten minutes for the participant to rest. Another way to ensure the independence of these two tests or sessions is to take different baseline $\mathrm{StO} 2$ for the two tests. Right before the emotional stress and physical stress, the HSI data was recorded to generate emotional stress baseline StO2 (ES_baseline in Figure 1) and physical stress baseline (PS_baseline in Figure 1). Right after the stress test, the HSI data was recorded again to generate emotional stress StO2 (ES in Figure 1) and physical stress StO2 (PS1 and PS2 in Figure 1). The differences between baseline and stress StO2, ES-ES_baseline and PS-PS_baseline, were used to classify the two types of stress.

\subsection{Processing of HSI Data}

The processing flow of HSI data is shown in Figure 2. The raw HSI date of faces were processed by using Modified Beer Lambert Three-Chromosphere model $[15,24]$ to obtain facial StO2 data. Because each pixel of the $\mathrm{StO} 2$ image is affected by surrounding tissues, the final $\mathrm{StO} 2$ image was generated by averaging the raw $\mathrm{StO} 2$ image. For the convenience of subsequent use, we then registered the acquired facial $\mathrm{StO} 2$ image to the standard face through 65 key points of the face.
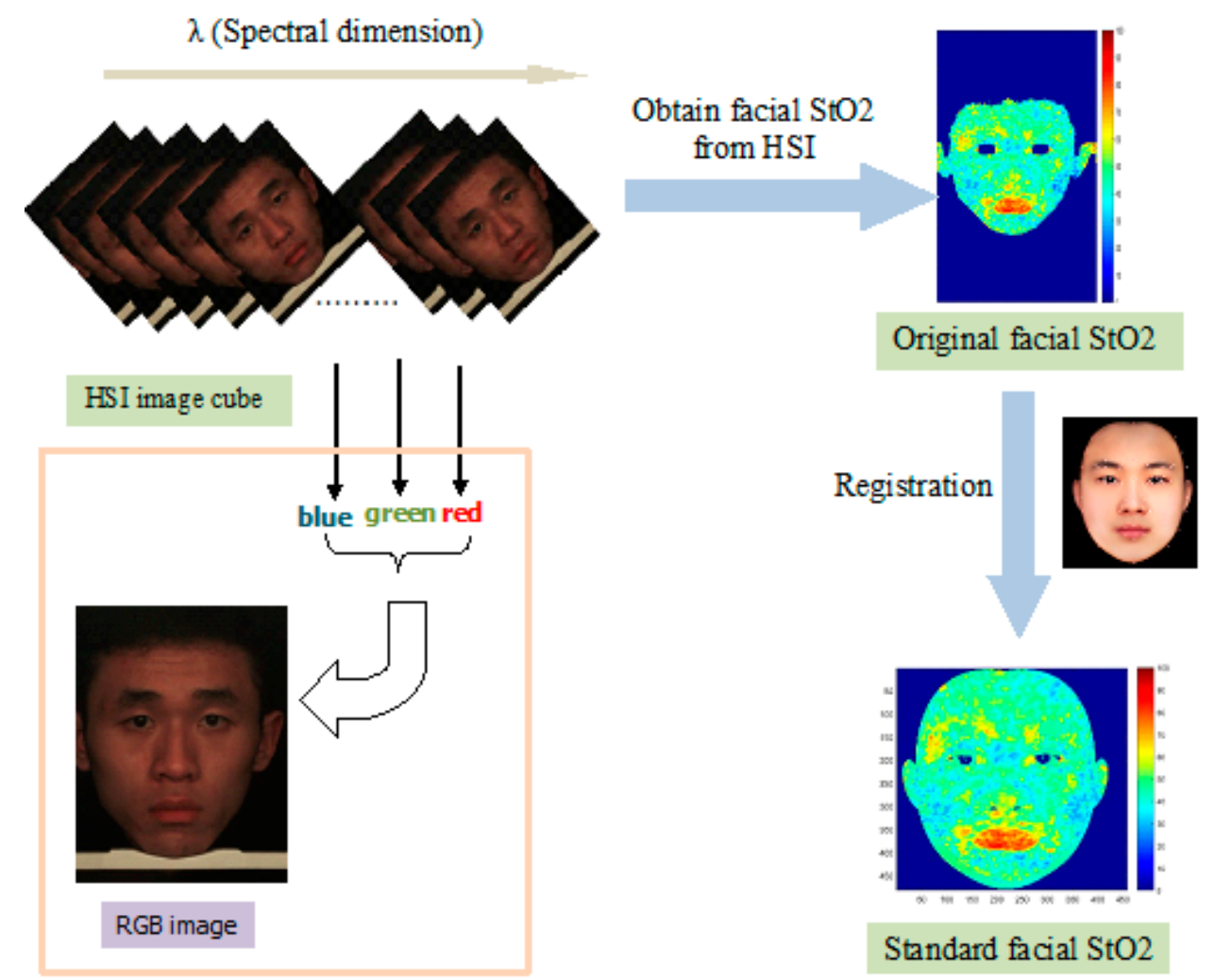

Figure 2. Processing flow of HSI data. The box in the lower left part indicates that R, G and B bands can be extracted from the HSI cube to form an RGB image.

The standard face (see Figure 3a) is the average image of 100 students' face images from Southwest University, which is generated by superimposing and averaging these 100 images through key points of the face. The $\mathrm{StO} 2$ registration process is as follows: Firstly, according to the facial 65 key points (see Figure $3 b$ ), we select the corresponding points on the original face image one by one to form a $65 \times 2$ matrix. Then, through geometric transformation, the original matrix is mapped to the same size 
matrix of 65 key points on the standard face, and a fourth-order polynomial transformation matrix is obtained. Finally, through this fourth-order polynomial transformation matrix, the original $\mathrm{StO} 2$ can be registered to the standard face.
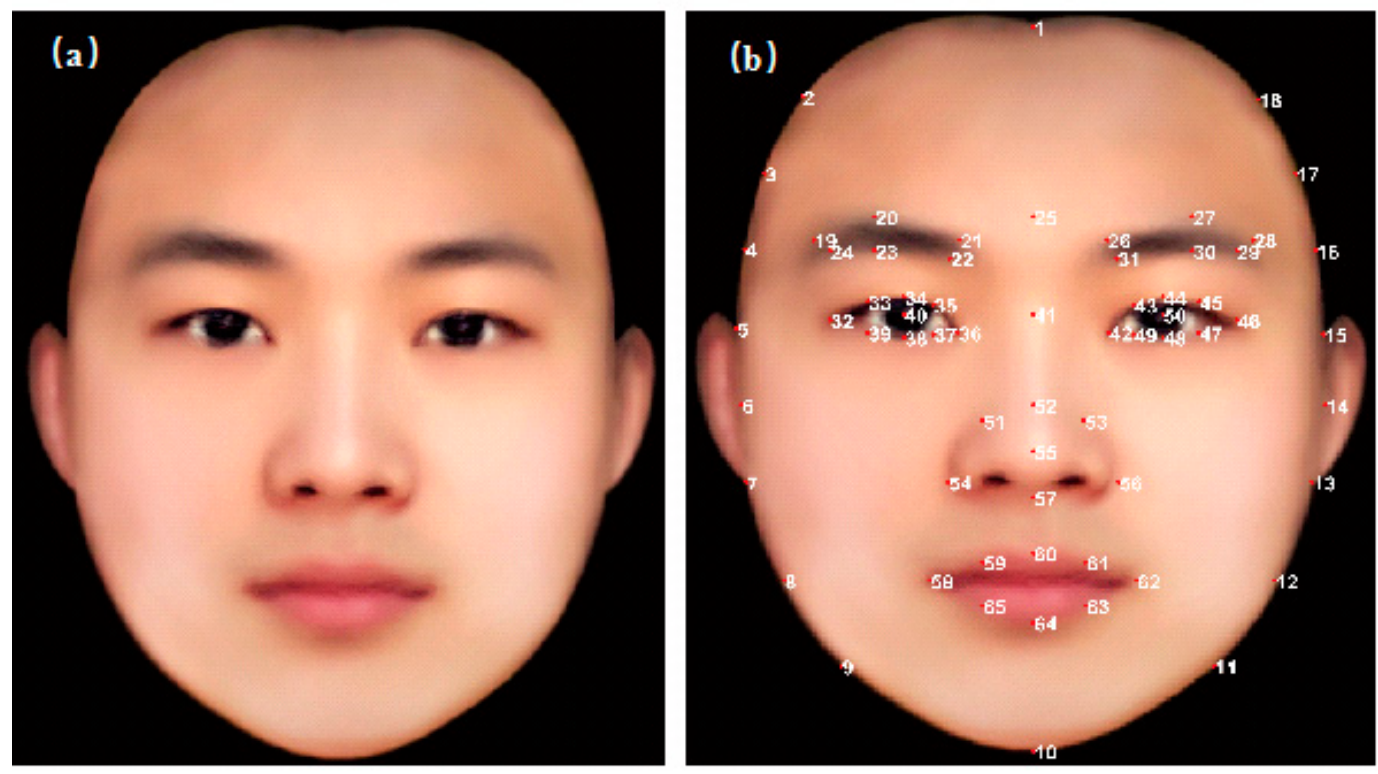

Figure 3. (a) The average standard face of 100 face images. (b) 65 facial key points for registration.

By performing this processing, all the $\mathrm{StO} 2$ faces of different shape will become the same shape, so that the regional facial $\mathrm{StO} 2$ analysis can be conducted precisely (the size of the $\mathrm{ROI}$ of every $\mathrm{StO} 2$ face is the same). In our previous pilot study [21], we registered the face through 23 key points. In this paper, we used more key points for registration to achieve higher accuracy.

The ear region is not used in this research, therefore the key points do not include the points on the ear. The $\mathrm{StO} 2$ values in the final registered $\mathrm{StO} 2$ maps in ear regions are average $\mathrm{StO} 2$ values from the neighbor face regions and do not indicate real $\mathrm{StO} 2$ values of the ears.

\subsection{Overview of StO2 Stress Database}

The StO2 Stress database (Table 1) include stress data from 42 participants. Data for each participant include emotional stress data ES, physical stress data PS1, PS2, emotional stress baseline data ES_baseline, and physical stress baseline data PS_baseline. The data is facial StO2 image after registration, with the size of $513 \times 911$. Therefore, the StO2 Stress database include $210(42 \times 5)$ facial $\mathrm{StO} 2$ images. The baseline data can be used to remove individual differences. It is worth noting that due to the nature of the HSI imaging system mentioned earlier (see Section 2.2), some raw $\mathrm{StO} 2$ images may be distorted and stretched. However, after registration this distortion does not affect the subsequent analysis and use of data.

Table 1. Overview of StO2 stress database.

\begin{tabular}{cccc}
\hline $\begin{array}{c}\text { Total Number of } \\
\text { Participants }\end{array}$ & Stress Categories & $\begin{array}{c}\text { Spatial Resolution of } \\
\text { Each Image }\end{array}$ & $\begin{array}{c}\text { Total Number of StO2 } \\
\text { Images }\end{array}$ \\
\hline & Baseline of ES & & \\
ES & Baseline of PS & $513 * 911$ & 210 \\
& PS1 & & \\
\hline
\end{tabular}




\section{Facial StO2 of Stress}

In this research, seven regions of interest (ROIs) were selected to explore the changes of $\mathrm{StO} 2$ under different types of stress, i.e., forehead [15], left cheek, nose, right cheek, chin, the middle of the eyebrow (meixin) [16,21], and philtrum [16,21]. Within these ROIs, two [16,21] of them have been investigated under solo type of stress. The rest five will be examined for the first time in this paper. The seven regions selected cover most of the upper, middle and lower parts of the face, which may fully represent facial $\mathrm{StO} 2$ features. The selected ROIs and their numberings are shown in Figure 4 . Among them, ROI1 represents forehead, ROI2 represents the right cheek, ROI3 represents the nose, ROI4 represents the left cheek, ROI5 represents the chin, ROI6 represents the philtrum and ROI7 represents the meixin. Because all the $\mathrm{StO} 2$ maps were registered in a standard face, the area of each ROI of each participant is the same. When the HSI camera took a face image, the left part of the image collected was actually the right part of the participant, and no image mirror flip was performed in the subsequent processing. So ROI2 represents the right cheek of the participant, and ROI4 represents the left cheek of the participant. The mean StO2 (M_StO2) value within each ROI calculated. In the study of facial $\mathrm{StO} 2$ changes under different stress states, we selected typical data of six participants for analysis. Of course the $\mathrm{StO} 2$ figures of all the 42 participants can be accessed in our published database. The data of the six participants selected are named from sub1 to sub6, and the corresponding ID of these participants in the database is $2,15,19,10,23$ and 29 , respectively.

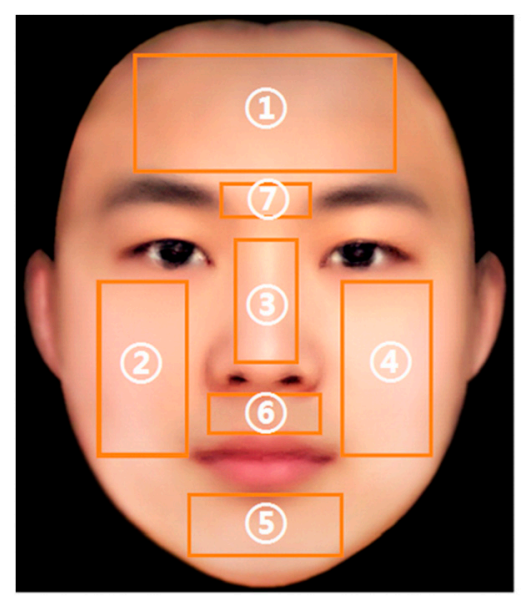

Figure 4. Illustration of seven selected ROIs.

\subsection{Facial StO2 of Emotional Stress}

The left part of Figure 5 shows the facial StO2 of six participants under baseline state and ES state, and right part shows the delta\% of ES StO2, with the warm color representing high $\mathrm{StO} 2$ value and high delta\% (see color bar of Figure 5). Figure 6 shows the average StO2 values within ROIs under baseline state and emotional stress state. From Figure 5, it is seen that the ES facial StO2 are higher than the baseline facial StO2 visually (more warm color is presented). Quantitative analysis of change of StO2 due to ES from Figure 6 indicates that $\mathrm{StO} 2$ rises in the forehead region under emotional stress, which is consistent with our previous research [15]. Meanwhile, the left and right cheeks and the meixin region (except for sub 4) also rise significantly. However, compared to other regions, the increase of $\mathrm{StO} 2$ in the nose region was small or even unchanged (see Figure 6). $\mathrm{StO} 2$ in the chin region even decreased in three of these six participants (sub3, sub5, sub6). 




(a) baseline

(b) ES
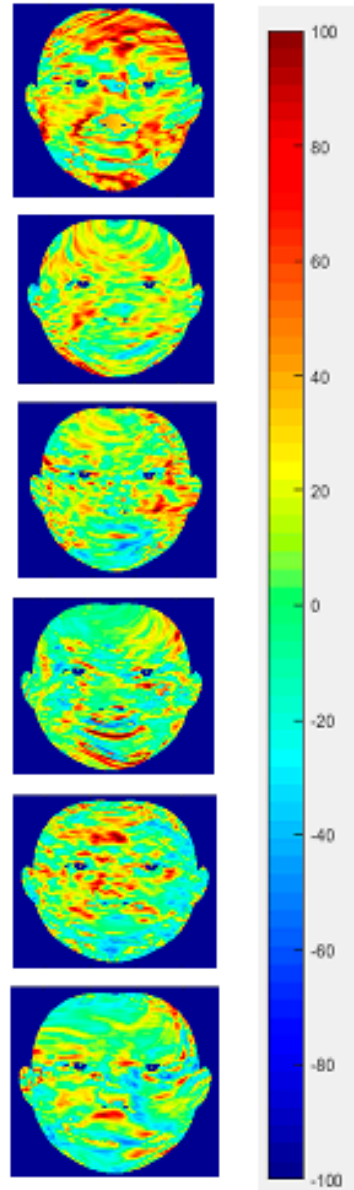

(c) delta $\%=\left(\right.$ ES-baseline)/baseline ${ }^{*} 100$

Figure 5. Facial StO2 maps of six participants under (a) baseline and (b) emotional stress. (c) delta $\%$ represents the incremental percentage of facial StO2 under emotional stress.

The average $\mathrm{StO} 2$ of every $\mathrm{ROI}$ of all participants can form a vector with 42 elements (42 participants). Performing statistical analysis on this vector (maximum, minimum, median, quartile), we can obtain a boxplot shown in Figure 7. It is observed that the ES StO2 are higher than baseline $\mathrm{StO} 2$ statistically. However, the $\mathrm{StO} 2$ within $\mathrm{RIO} 3$ (nose), ROI5 (chin), and ROI6 (Philtrum) have smaller change. At the same time, we calculate the delta score of M_StO2=ES-ES_baseline in seven ROIs of all participants under emotional stress, which are illustrated in Figure 8 with colors representing facial regions, numbers at horizontal axis representing ID of participants, and numbers in vertical axis representing delta score of M_StO2. It can be seen that most of the delta scores are larger than 0, which indicates that the ES StO2 trend is rising compared to the baseline. The delta scores that are smaller than 0 mostly belong to chin (black dots in Figure 8) and philtrum (green dots) areas, and the delta scores of nose areas (gray dots) are mostly near the 0 , which indicate that the nose, chin and philtrum area will have smaller rising. These observation accords with the boxplot in Figure 7. 


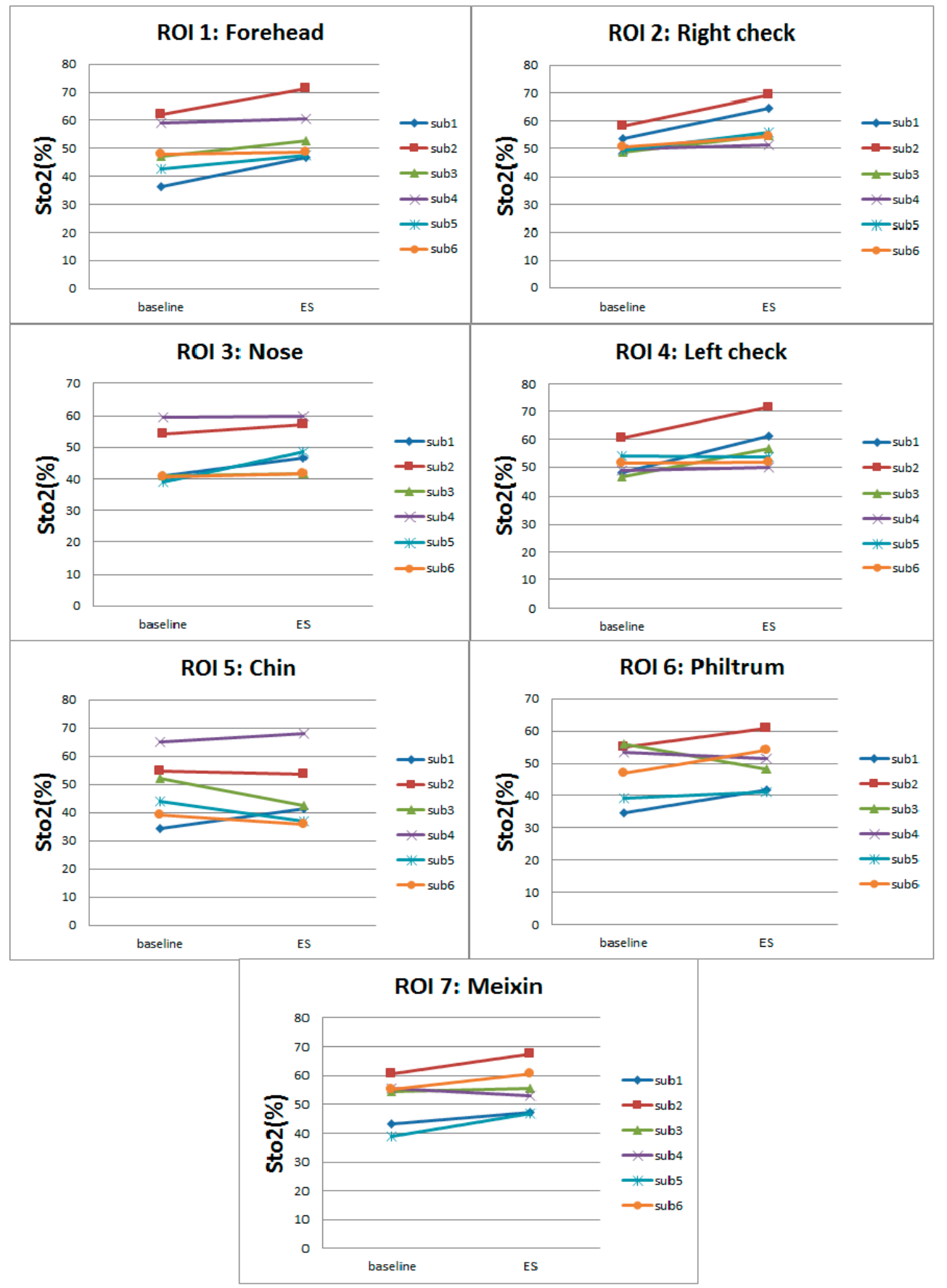

Figure 6. Average facial StO2 value of seven ROIs under baseline and emotional stress. 


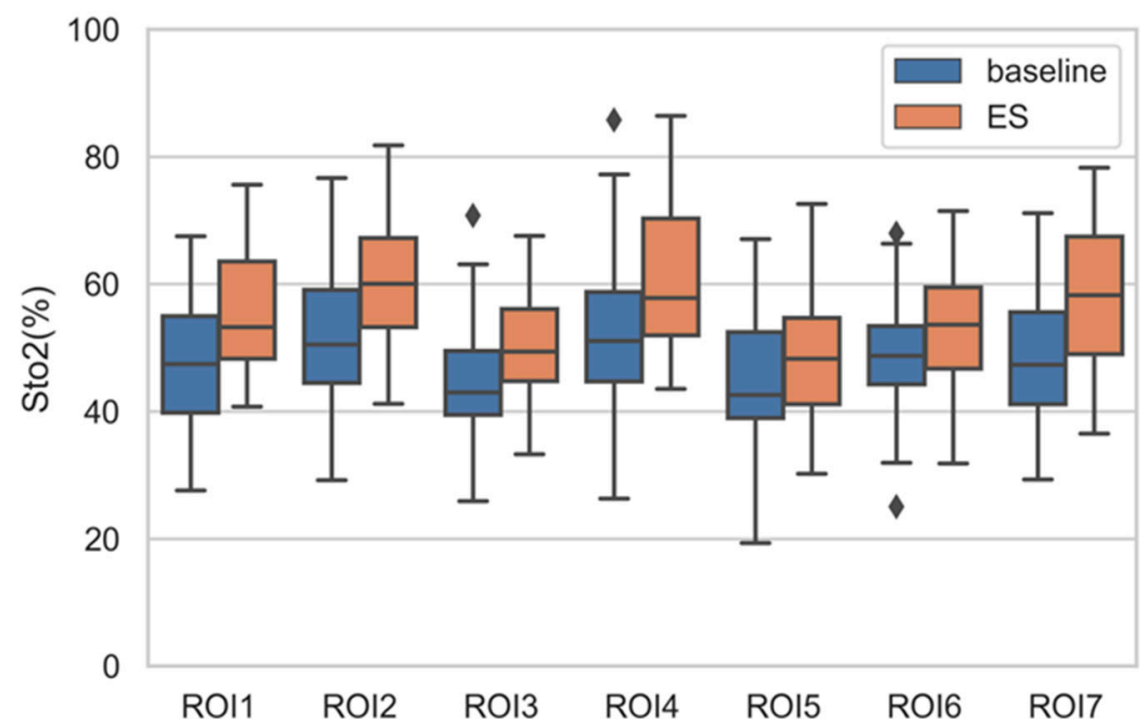

Figure 7. Boxplot of average StO2 of ROIs of all participants under ES and baseline.

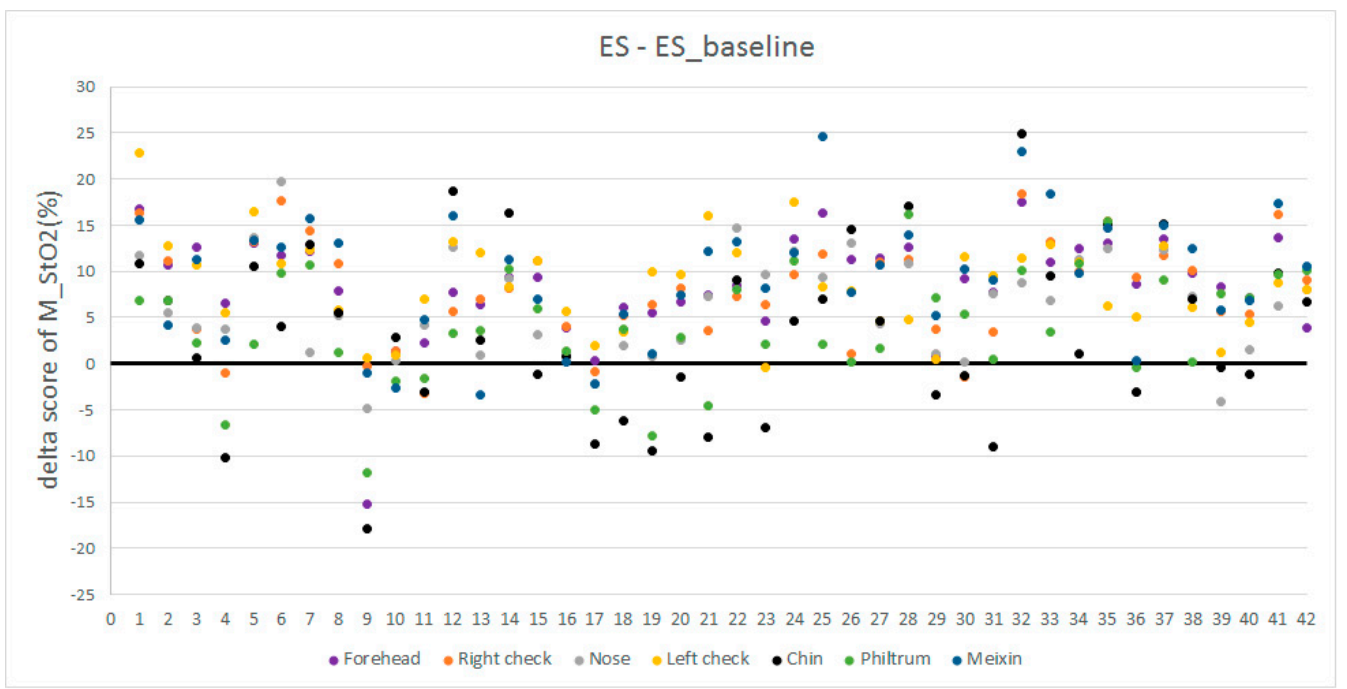

Figure 8. Delta score of M_StO2=ES-ES_baseline.

The increase of facial StO2 during ES could be due to the "fight-or-flight" state. When a person is under emotional stress, this "fight-or-flight" state could be triggered. The brain needs to function more quickly to prepare for the potential threat and the eye needs to see more sharply [36], therefore more oxygenated blood could flow into head through the neck artery. This oxygenated blood also floods the face and makes most of the facial areas experience higher StO2. The chin, philtrum, and nose areas are relatively small areas compared to the other facial areas. Most of lower StO2 during ES are observed in these three areas. We do not have a good physiological explanation for this observation. The small cases of lower $\mathrm{StO} 2$ in this area might be due to individual differences in the blood flow distribution mechanism. During the ES, the blood vessels of these areas are contracted and the blood flow to these three areas is restricted to supply more oxygenated blood to the more functional areas such as the eyes and brain. This phenomenon is similar to the "cold nose during stress". When some persons are stressed, the blood flow to the nose can be reduced and thus they feel a cold nose.

\subsection{Facial StO2 of Physical Stress}

In our pilot experiment [21], in which 20 participants took physical stress tests, we found that some had lower overall PS1 data than baseline, while others had higher PS1 data. After a two-minute 
rest, the PS2 gradually rose towards baseline. In this paper, we increased the number of participants to 42 , and still observed this phenomenon.

The left part of Figure 9 shows the facial StO2 of the six participants under baseline, PS1, and PS2 states, and right part shows the delta \% of PS1 StO2 and PS2 StO2, respectively. Figure 10 shows the average $\mathrm{StO} 2$ values within ROIs under different states. It is seen from Figure 9 that three participants, i.e., sub1, sub2, sub3, had lower PS1 StO2 than the baseline, while for sub4, sub5, and sub6, their PS1 $\mathrm{StO} 2$ was higher than the baseline. These conclusions are reflected in the delta\% (Figure 9d) that the most delta\% colors of PS1 StO2 of sub1, sub2, sub3 are cold colors, while the most delta\% colors of sub4, sub5, and sub6 are warm colors. Quantitative analysis from Figure 10 confirms the observation of Figure 9. All the PS1 StO2 values in the seven ROIs are lower than baseline in the three participants (left column of Figure 10). For the other three participants, forehead, nose, cheek (except for right cheek of sub5) all experienced little higher PS1 StO2.

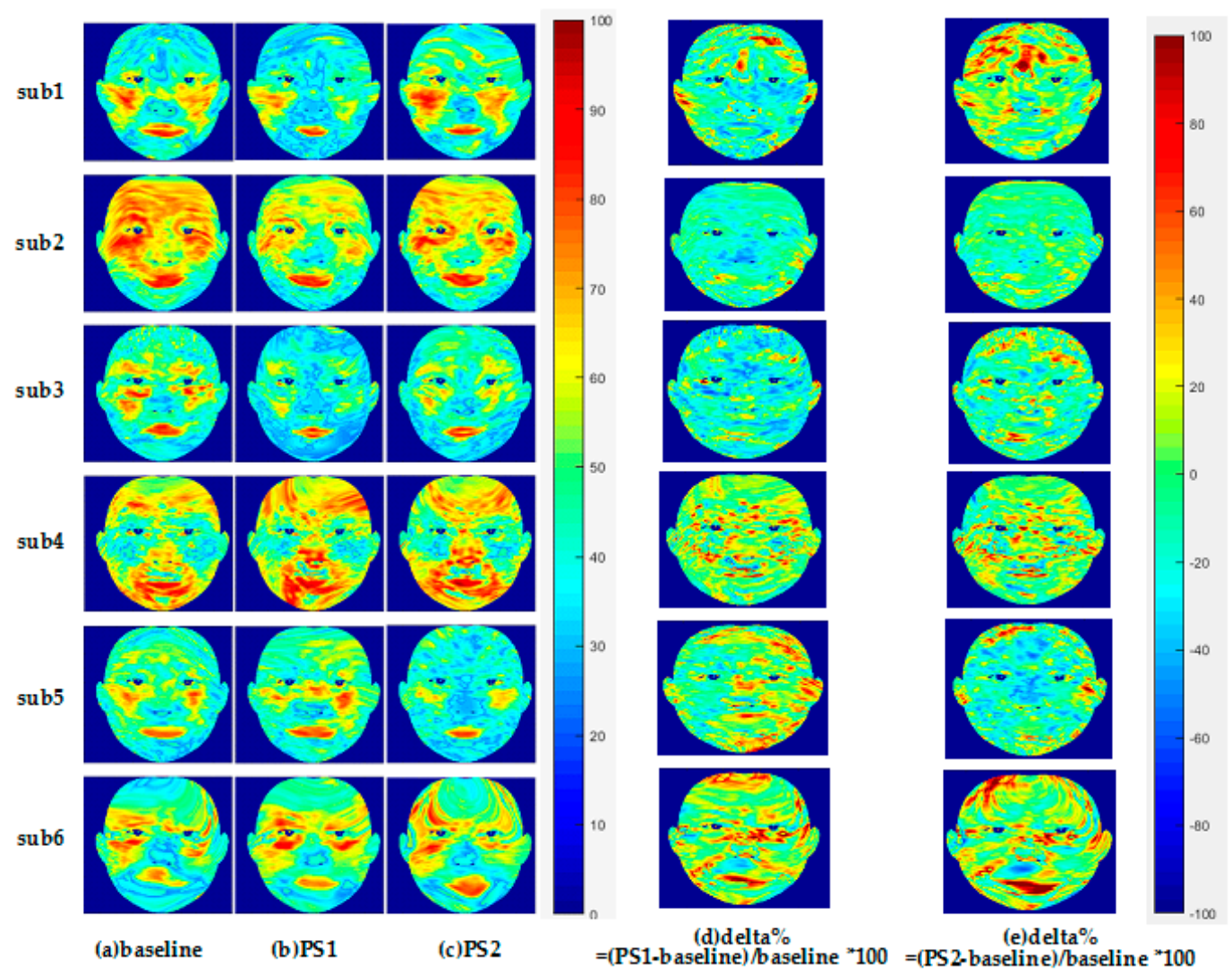

Figure 9. (a-c) Facial StO2 maps of six participants under physical stress. PS1 StO2 of the first three participants are lower than baseline $\mathrm{StO} 2$. (d,e) delta\% represents the incremental percentage of facial $\mathrm{StO} 2$ under physical stress. 




Figure 10. Average facial $\mathrm{StO} 2$ value of seven ROIs under baseline and physical stress. The left column represents PS1 StO2 lower than baseline StO2.

By analyzing all 42 subjects, we found that more than $60 \%$ of the participants had lower PS1 StO2 than the baseline StO2. After a two-minute rest, the PS2 increases from PS1, which means PS2 changes towards the baseline value. On the other hand, other participants showed an overall increased PS1 
compared to the baseline. The PS2 of these participants decreased compared to PS1, which means the PS2 also changes towards baseline. The boxplots of average StO2 of ROIs of all participants under PS and baseline are shown in Figure 11. The average PS1 StO2 is lower than average baseline StO2 in all ROIs. This is because more than $60 \%$ participants have lower PS1 StO2. The PS2 StO2 are all higher than PS1 and recover towards baseline.

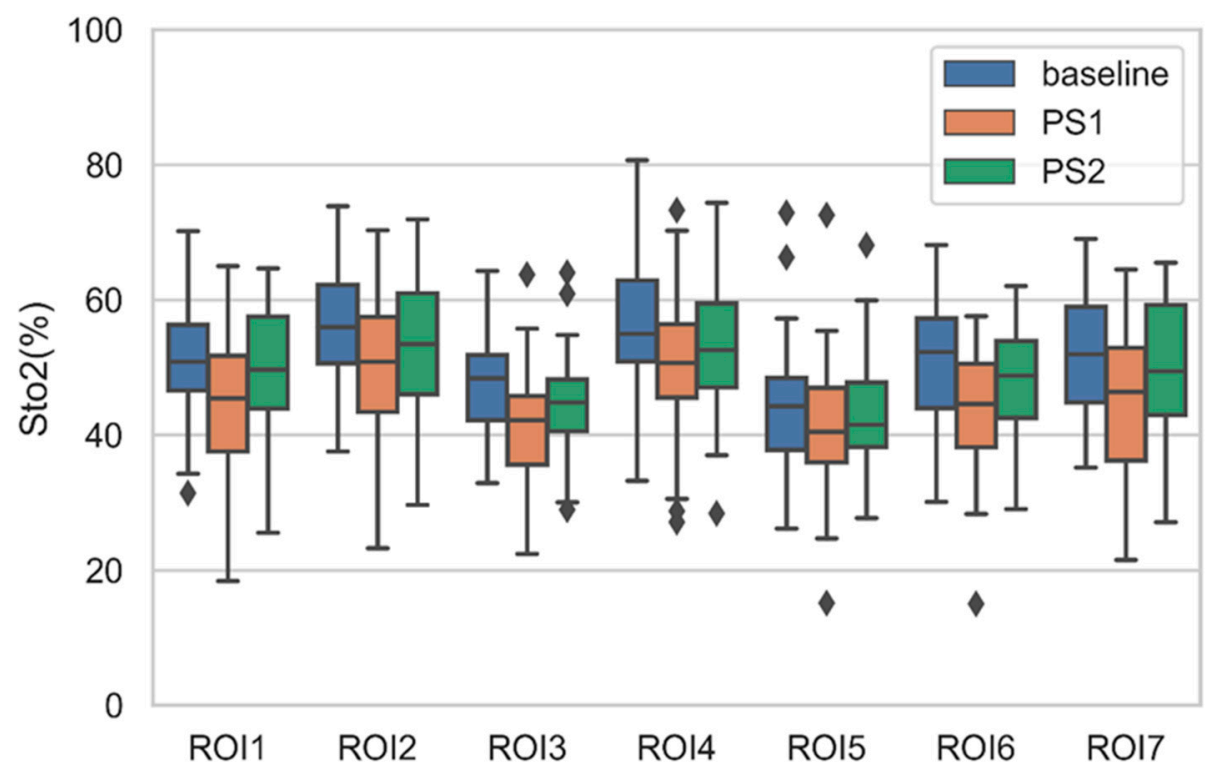

Figure 11. Boxplot of average $\mathrm{StO} 2$ of ROIs of all participants under PS and baseline.

$\mathrm{PS} 1 \mathrm{StO} 2$ is the $\mathrm{StO} 2$ collected when physical stress just finished, while $\mathrm{PS} 2 \mathrm{StO} 2$ is the $\mathrm{StO} 2$ collected when physical stress gradually dropped but had not completely disappeared. The different change trends of PS1 StO2 may thus be understandable. A familiar scene similar to this could be that different people may have different facial color changes right after a thousand-meter race. Some will turn pale and others will turn red. This difference may be due to the diversity of blood regulation mechanisms of individuals. When some persons exercise vigorously, the hearts beat faster and the breathing become faster. At this time, the cells of the body need a lot of oxygen support, which makes the blood vessels dilate and faces become red. However, for some other individuals, the ability of blood to carry and transport oxygen might be limited. When these people do exercise, most of the blood is supplied to the muscles, which causes the blood to the face to be reduced, resulting in the appearance of facial paleness.

We also calculated the delta scores of M_StO2 = PS1-PS_baseline, M_StO2 = PS2-PS1, and M_StO2 = PS2-PS_baseline in seven ROIs of all participants, which are illustrated in Figure 12 with colors representing facial regions, numbers at horizontal axis representing ID of participants, and numbers in vertical axis representing M_StO2. It is seen in Figure 12 that most data of M_StO2 = PS1-PS_baseline are smaller than 0, which confirms that the PS1 is lower than PS_baseline in most of the cases. For PS2-PS1, more than half of the values are larger than 0 . The participants with more dots over 0 line in the PS1-PS_baseline (upper part of Figure 12) will have more dots below 0 line (middle part of Figure 12). For example, the participants 10, 29, and 33 have more dots over black line (0 values) in the PS1-PS_baseline plot, they also have more dots below the black line in the PS2-PS1 plot. This indicates that for those participants having higher PS1 than baseline, they will have lower PS2 than PS1, and their PS2 StO2 is changing towards baseline. This is also true for those participants with lower PS1 than PS_baseline, their PS2 are also changing towards baseline. By observing the PS2-PS_baseline in the lower part of Figure 12, we can see that more than $60 \%$ of the dots deviate more than $5 \%$ from the 0 value, which indicates that most participants still do not recover to baseline after 2 min rest. But comparing to the PS1-PS_baseline, the dots in PS2-PS_baseline are closers to the 0 value. 
There is no particular $\mathrm{StO} 2$ changing pattern, like that in the ES test, observed for specific facial areas in the PS test.
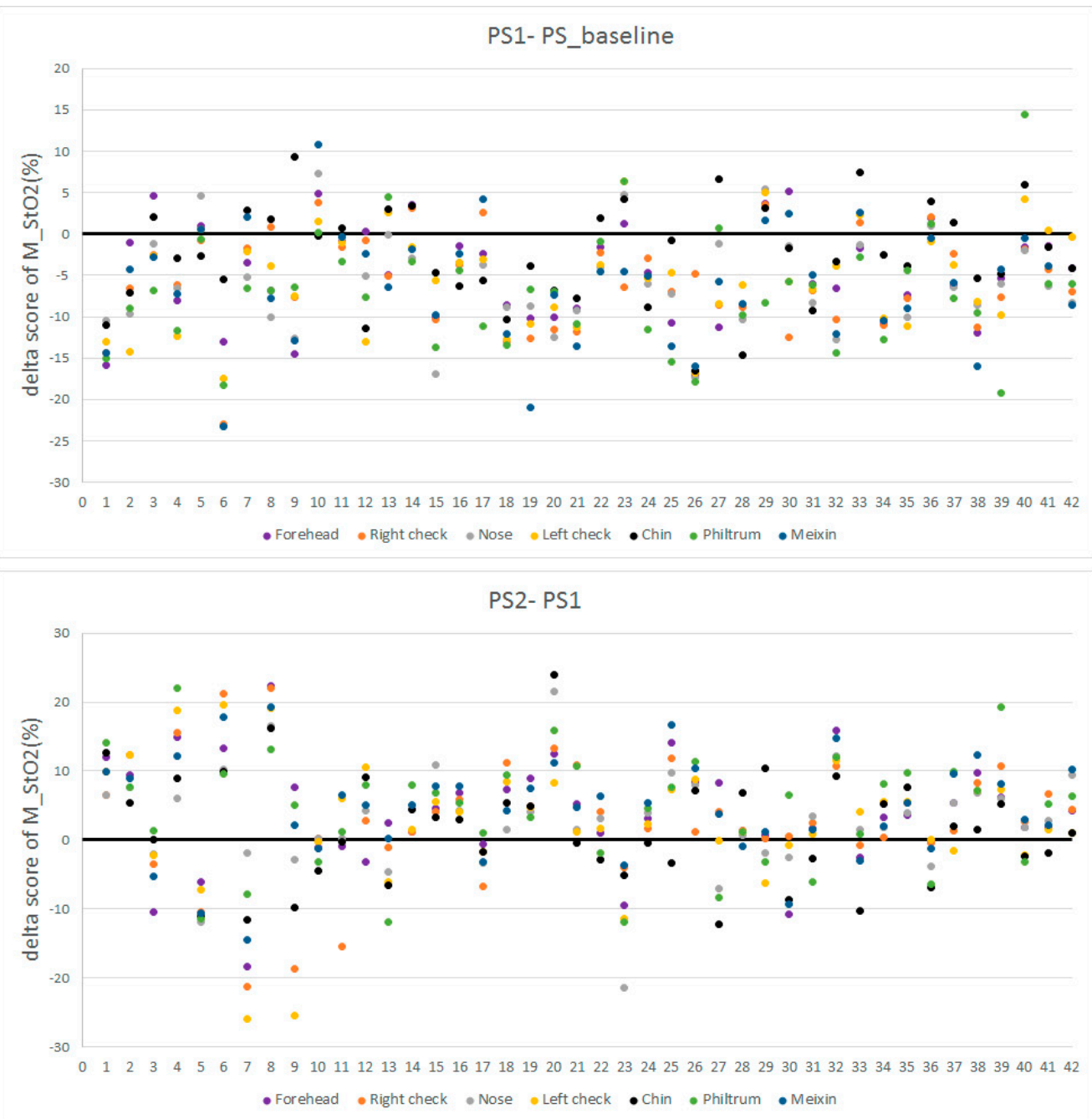

PS2- PS_baseline

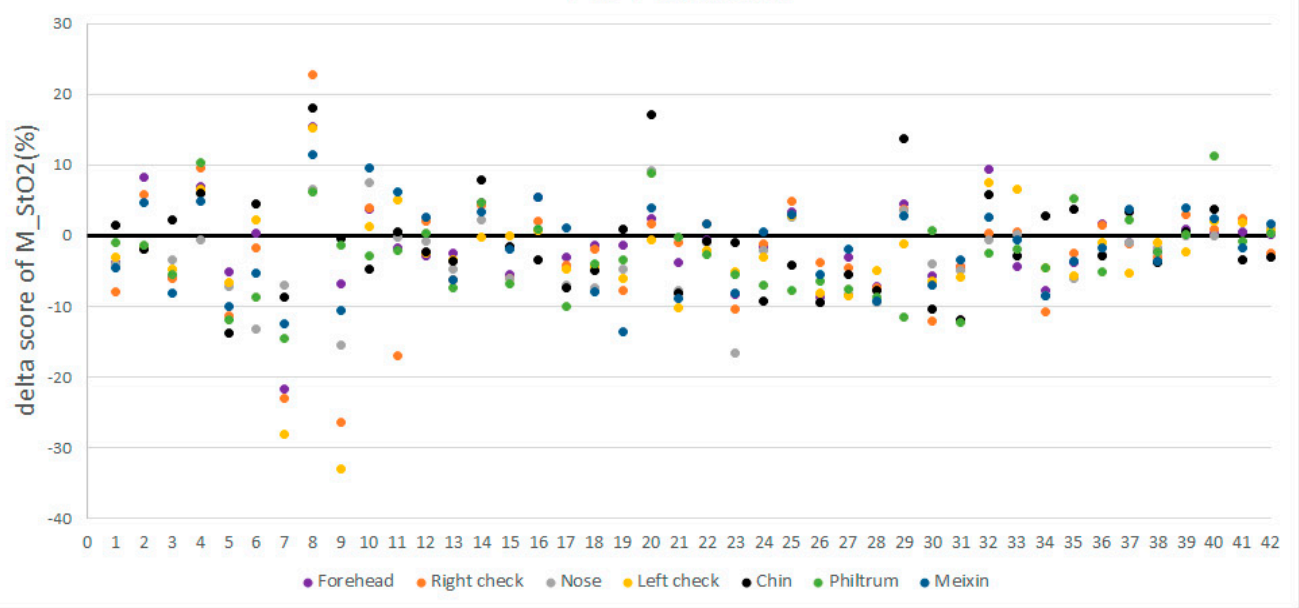

Figure 12. Delta score of M_StO2 = PS1-PS_baseline, M_StO2 = PS2-PS1 and, M_StO2 = PS2-PS_baseline. 


\section{Stress Detection}

\subsection{Feature Extraction}

Based on the analysis of ROI StO2, it is obvious that the value of $\mathrm{ROI} \mathrm{StO2} \mathrm{could} \mathrm{be} \mathrm{an} \mathrm{indicator}$ of stress. However, it is hard to determine which ROI or which combination of ROIs could be used to classify the two types of stress only by using statistic methods. Since there are seven selected ROIs, the number of the combination of ROIs is $7+21+35+35+21+7+1=127$ (which is not large), we used an exhaustive search to find the best combination of ROIs that can give the highest classification rate.

For all participants, we took ES as the emotional stress data and PS1 as the physical stress data. The M_StO2 of ROIs were calculated as the features. StO2 may vary individually, it is influenced by adipose tissue thickness and gender [37]. Baseline information for each participant was also taken into account due to the individual differences among participants. The final features for the classification were M_StO2 of ES or PS1 minus M_StO2 of its corresponding baseline. Because each ROI produces one features, there are a total of 127 feature combinations (same as the number of ROI combinations). Each of 127 feature combinations was tried as input to a SVM classifier for the classification. The radial basis function (RBF) [38] was selected in SVM.

\subsection{Feature Selection}

Five-fold random cross-validation for 50 times was used in the classification task. The classification in this research is a two-class classification (either ES or PS). The database is well balanced, i.e., the number of ES samples is the same of the number of PS samples. Therefore, the classification rate is used as the object function, which is defined as the ratio of the number of predicted class to the number of actual class.

When the feature dimension is one (only one ROI), we can observe the effect of each ROI on the classification task. The average classification rate of every ROI is shown in Figure 13a. It is seen that the chin region (ROI5) had a poor effect on the discrimination of both stresses. The chin area only achieved an average recognition rate of $62.89 \%$ while the rest ROIs achieved an average recognition rate of more than $80 \%$.

Figure $13 \mathrm{~b}$ shows the average classification rate under different dimension size of features. When the feature dimension is 6 , there are seven combinations of features, i.e., ROI $\{1,2,3,4,5,6\}$ ROI $\{1,2,3,4,5,7\}$, ROI $\{1,2,3,4,6,7\}, \operatorname{ROI}\{1,2,3,5,6,7\}$, ROI $\{1,2,4,5,6,7\}$, ROI $\{1,3,4,5,6,7\}$, and ROI $\{2,3,4,5,6,7\}$. The classification rate at feature dimension 6 in the Figure $13 \mathrm{~b}$ is the average classification rate of these seven cases, which is $91.61 \%$. It is observed from Figure $13 \mathrm{~b}$ that as the dimension of features increases, the average of classification rate increases.

Figure 13c shows the best classification rate and combination of features within each feature dimension size. It is seen that the best classification rate at dimension size 6 is achieved by the combination ROI $\{1,2,3,4,5,7\}$, which is $93.09 \%$. The highest classification rate $(95.56 \%)$ of all 127 features combinations is achieved by combination ROI $\{4,5,7\}$.

Therefore, the selected ROIs/features are left cheek (ROI4), chin (ROI5) and meixin (ROI7). These three ROIs correspond to the middle, lower and upper facial areas of the whole face, so it is understandable that this combination can achieve a high recognition rate.

\subsection{Cheek ROIs Comparison}

The three selected ROIs correspond to the middle, lower and upper facial areas of the face. The middle part is the left cheek (ROI4). Because the blood vessels on both cheeks are symmetrical [39], it is natural to ask whether right cheek (ROI2) will have the same effect if it replaces the left cheek. The classification rates achieved by ROI4 and ROI2 are compared in Table 2 . ROI4 can give $2 \%$ higher classification rate than ROI2 in both ES and PS situation. This finding might be related to the theory of division of labor between right and left brains [40-43]. 
(a)



(b)



(c)

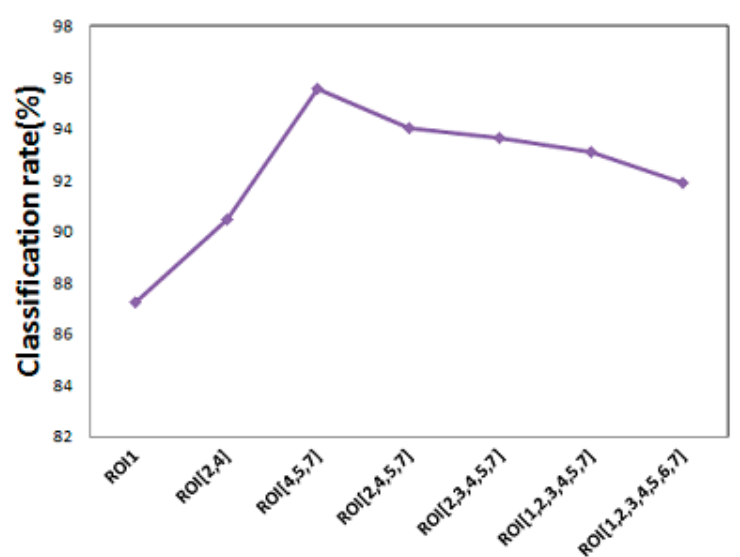

Figure 13. ROI combinations and classification rate. (a)The classification rate of every single ROI. (b)The average classification rate of combinations of ROIs in seven feature dimensions. (c) The maximum classification rate of combinations of ROIs in seven feature dimensions.

Table 2. Comparison of accuracy between ROI2, ROI4 and feature combination with ROI5 and ROI7.

\begin{tabular}{cccc}
\hline Single Feature or Combination of Features & ES (\%) & PS (\%) & Average (\%) \\
\hline ROI2 & 84.71 & 84.61 & 85.66 \\
ROI4 & 87.26 & 86.78 & 87.02 \\
ROI $\{2,5,7\}$ & 85.78 & 91.94 & 88.86 \\
ROI $\{4,5,7\}$ & 96.82 & 94.3 & 95.56 \\
\hline
\end{tabular}

Sperry's split brain experiment in the late 1950s found that for the vast majority of right-handed people and the majority of left-handed people, the left hemisphere was associated with verbal, reasoning, rational and analytical thinking, while the right hemisphere was associated with perceptual, spatial subject perception and intuitive thinking. Therefore, the left brain could be called "conscious 
brain", "academic brain" and "language brain", while the right brain could be called "subconscious brain", "creative brain", "musical brain" and "artistic brain". In our experimental process, stimulus sources of ES include timed mental calculation and Stroop Color-Word tests, both of which require rational calculation and analysis of participants. According to Sperry's theory of the right and left brain, during the experiment of ES, the left brain activity increased, and the StO2 in the left cheek connected with the left brain nerve increased, resulting in the left cheek (ROI4) features showing considerable superiority to the identification of ES. In order to explore whether the StO2 in the left cheek (ROI4) was significantly higher than that in the right cheek (ROI2) under the ES condition, we performed a $t$-test.

However, the $t$-test results between $\mathrm{StO} 2$ of ROI2 and ROI4 under various affective states in Table 3 show that there is no statistical difference between these two areas. The values that we used to conduct the $t$-test are the features that we used to detect stress. The $h$ in Table 3 is the result of test, where $h=0$ means there is no statistical difference between two datasets and $h=1$ means there is a difference. The $p$ is possibility. The smaller the $p$ value, the more the difference. In general, when $p$ is less than 0.05 , we believe that there is a statistical difference between the two datasets. The $c i$ is confidence interval and the $t$ is the $t$-test value. The results of the $t$-test directly indicate that the difference of $\mathrm{StO} 2$ changes in the left and right cheeks could not reach statistical significance under neither emotional stress nor physical stress. The observation in this research might also be because that the dataset is not large enough, the division theory does not apply in our study.

Table 3. T-test results of ROI2 and ROI4 features.

\begin{tabular}{llccc}
\hline Features for $\boldsymbol{t}$-Test & $\boldsymbol{h}$ & $\boldsymbol{p}$ & $\boldsymbol{c} \boldsymbol{i}$ & $\boldsymbol{t}$ \\
\hline All_ROI2,All_ROI4 & 0 & 0.3776 & $-1.6550,0.6340$ & -0.8872 \\
ES_ROI2,ES_ROI4 & 0 & 0.4111 & $-2.4781,1.0340$ & -0.8304 \\
PS_ROI2,PS_ROI4 & 0 & 0.6973 & $-1.8402,1.2423$ & -0.3917 \\
\hline
\end{tabular}

We have also examined the combination of $\mathrm{ROI}\{2,5,7\}$ and found this combination can only give a classification rate of $88.86 \%$, much lower than what ROI $\{4,5,7\}$ can achieve $(95.56 \%)$. This observation from purely data analysis confirm that the left cheek is more suitable for stress detection in terms of achieving higher accuracy. The superiority of ROI $\{4,5,7\}$ may came from the combination of ROI4 and the remaining two ROIs.

\subsection{Classification of PS and ES}

We used optimal ROI feature combination as input to some classical machine learning algorithms to classify the ES and PS, and the classification results were shown in Table 4. Except for the decision tree, all the classification rate achieved were more than $90 \%$. In other words, the feature combination of the left cheek (ROI4), chin (ROI5), and meixin (ROI7) is robust for stress detection. All of the above results also indicate that stress can be identified and that the non-contact method of extracting $\mathrm{StO} 2$ features through HSI can effectively separate emotional stress from physical stress.

Table 4. Recognition rate of other algorithms with the best ROIs' features as the input.

\begin{tabular}{cc}
\hline Algorithms & Accuracy \\
\hline Linear Discriminant & $90.5 \%$ \\
Logistic Regression & $91.98 \%$ \\
KNN & $92.28 \%$ \\
Decision Tree & $86.9 \%$ \\
Ensemble learning & $92 \%$ \\
\hline
\end{tabular}

\subsection{Comparison with Other Methods}

Since there was no public HSI stress database before, there are no studies on using HSI to classify stress. Therefore, we cannot directly compare our results with those of others. However, we can 
compare achieved accuracy with that of the other methods, which have the similar goal with our task. The comparison is divided into three parts: the first part is the comparison with emotional stress detection, as shown in Table 5; the second part is the comparison with physical stress detection, as shown in Table 6; the third part is the comparison with the classification of emotional stress and physical stress, as shown in Table 7.

In related researches of emotional stress detection, some researchers [44-46] used physiological signals collected by the contact method to perform stress detection. Non-contact stress detection $[14,15,20,47,48]$ had also achieved good results with the development of imaging technology. As shown in Table 5, among all methods, whether contact or non-contact, our proposed method achieved the highest accuracy $(96.82 \%)$. Moreover, the number of features in our method is very small (only three features).

Table 5. Comparison of emotional stress detection.

\begin{tabular}{|c|c|c|c|c|}
\hline Methods & Measurements & Amount of Features & Classifier & Accuracy \\
\hline [46] & Wearable EEG devices & 80 & Gaussian SVM & $80.32 \%$ \\
\hline [14] & $\begin{array}{l}\text { Five band DC for heart rate, } \\
\text { breath rate, HRV }\end{array}$ & 3 & Linear SVM & $85 \%$ \\
\hline [15] & HSI for StO2 signal of forehead & 1 & Binary classifier & $88.1 \%$ \\
\hline [44] & Galvanic skin response & 16 & ANOVA test & $89 \%$ \\
\hline [45] & ECG, HRV & 20 & KNN, SVM & $92.75 \%$ \\
\hline [47] & Thermal signals & 3 & EM-CCA, BP & $93.3 \%$ \\
\hline [48] & $\begin{array}{l}\text { Non-contact Bioradar for } \\
\text { respiratory signals }\end{array}$ & 3 & $\begin{array}{l}3 \text { layer } \\
\text { perceptron }\end{array}$ & $94.44 \%$ \\
\hline [20] & Kinect for respiratory signals & 56 & SVM & $94.76 \%{ }^{1}$ \\
\hline Ours & HSI for facial StO2 signals & 3 & SVM & $96.82 \%$ \\
\hline
\end{tabular}

${ }^{1}$ Results of classification for emotional stress and the other two states (physical stress and relaxation).

Table 6. Comparison of physical stress detection.

\begin{tabular}{ccccc}
\hline Methods & Measurements & Amount of Features & Classifier & Accuracy \\
\hline$[21]$ & HSI for facial StO2 signals & 5 & SVM & $82.11 \%$ \\
{$[47]$} & Thermal signals & 3 & Back Propagation & $93.3 \%$ \\
{$[16]$} & MSI for StO2 signal & 2 & MCDS, LSTM & $93.3 \%{ }^{1}$ \\
{$[20]$} & Kinect for respiratory signals & 56 & SVM & $92.17 \%^{2}$ \\
Ours & HSI for facial StO2 signals & 3 & SVM & $94.3 \%$ \\
\hline
\end{tabular}

${ }^{1}$ The result is the average accuracy of PS for three different loads. ${ }^{2}$ Results of classification of physical stress and the other two states (emotional stress and relaxation).

Table 7. Comparison of emotional and physical stress classification.

\begin{tabular}{ccccc}
\hline Methods & Measurements & Amount of Features & Classifier & Accuracy \\
\hline$[47]$ & Thermal signals & 3 & EM-CCA, BP & $93.3 \%$ \\
{$[20]$} & Kinect for respiratory signals & 56 & SVM & $97.93 \%{ }^{1}$ \\
Ours & HSI for facial StO2 signals & 3 & SVM & $95.56 \%$ \\
\hline \multicolumn{5}{c}{}
\end{tabular}

Compared with emotional stress detection, there are relatively few physical stress detection studies. Early physical stress detection mainly focused on drivers' physical fatigue while driving [1,11]. In recent years, most physical stress detection research uses non-contact imaging-based methods [16,20,21,47]. The comparison of PS detection is given in Table 6. Among all the methods, our proposed method achieved the highest accuracy $(94.3 \%)$ and the feature dimensions of our method are only three.

Very few studies have been performed to distinguish between emotional and physical stress. There are two papers that discriminate between emotional stress and physical stress. Both papers used a contactless measurement, which was similar to the task we did. Hong et al. [47] distinguished the two types of stress based on thermal signals. Our previous work [20] used respiratory signals based on a Kinect depth perception camera for the classification. The average accuracy of stress classification is shown in Table 7. The classification rate achieved in this paper is higher than that in [47], but lower 
than that in [20]. However, the dimension size used in this research is much smaller than that used in [20], but the same as that in [47].

\section{Discussion and Conclusions}

In this study, we proposed to classify stress by using facial StO2 extracted from HSI. The way used to acquire HSI signals is a contact free method. Facial StO2 has been proved to be an excellent physiological indicator to distinguish stress. When using $\mathrm{StO} 2$ features to detect stress, only a few features can achieve a high accuracy.

We organized the data collected during the experiment into a public $\mathrm{StO} 2$ stress database, which contains two common stress categories, emotional stress and physical stress. Most studies so far have focused only on one type of stress detection. Our open stress database will provide researchers with a data platform to study and detect both types of stress.

By visualizing $\mathrm{StO} 2$ changes in seven major facial regions under two stress states, we observed different trends of $\mathrm{StO} 2$ change in these seven regions and the changes were also different among different persons. This indicates that the $\mathrm{StO} 2$ feature can effectively distinguish between the two types of stress. Moreover, by comparing the facial StO2 immediately after physical stress and two minutes later, we found that the change trend of $\mathrm{StO} 2$ among different participants is different. Some people had higher StO2 levels than baseline after physical stress, while others had lower ones. This is consistent with the phenomenon in one of our pilot studies [21]. Through data statistics, we found that PS1 StO2 below the baseline $\mathrm{StO} 2$ accounted for more than $60 \%$ of the total cases. However, this result is based on the fact that the participants are all young university students. This situation might change if more participants of different ages or jobs were added. The effect of age or exercise capacity on the change trend of PS StO2 is still unknown at this stage.

We tried all the feature combinations of the seven selected ROIs to classify stress and found the feature combination that can achieve the highest recognition rate of $95.56 \%$, i.e., the left cheek, chin, and meixin. At the same time, it can be observed that under this optimal combination of features, if the left cheek feature was replaced by the right cheek feature, the classification rate will be greatly reduced. To understand this phenomenon, we conducted a series data analyses. It was found that there was no significant difference in $\mathrm{StO} 2$ between the left and right cheeks, and the accuracy difference came from the combination of features.

The StO2 can be influenced by adipose tissue and thus differs between males and females [37]. However, the effect of adipose tissue on $\mathrm{StO} 2$ has not been considered in this research. The input wavelengths of this study is from $518 \mathrm{~nm}$ to $580 \mathrm{~nm}$ [15]. In this wavelength range, the main chromophores are $\mathrm{Hb}, \mathrm{HbO} 2$, and melanin, which have been considered in our $\mathrm{StO} 2$ producing model [15]. Pure mammalian fat starts to absorb light from around $700 \mathrm{~nm}$ and reaches absorption peaks at around $920 \mathrm{~nm}$ [49]. Though considering the effect of adipose tissue will provide more accuracy in the research, we have not taken this effect into account due to the wavelength used in this research being below $700 \mathrm{~nm}$. The exact reading of $\mathrm{StO} 2$ in this research might therefore deviate slightly from the real reading. However, the input features to the classification model are the PS1-PS_basline, and ES-ES_baseline, which are the difference between stress $\mathrm{StO} 2$ and baseline $\mathrm{StO} 2$. That is to say, we depend on the change of $\mathrm{StO} 2$ not the exact reading of $\mathrm{StO} 2$ to classify the two types of stress. This operation could avoid the effect of the small deviation of $\mathrm{StO} 2$ readings, because since the deviation due to the adipose tissue for the same person is fixed, the difference between stress state and baseline states can eliminate the deviation.

The StO2 was produced by using HSI in this research. The HSI [22] is also a spectroscopy technique, and a complementary imaging option of the point/line spectroscopy. It uses reflectance or transmittance at every wavelength of materials to analyze the contents of the materials. This characteristic is the same as that of NIR spectroscopy. However, the HSI normally takes a wider range of light wavelengths as the input, such as visible to near-infrared (from roughly $380 \mathrm{~nm}$ to $2500 \mathrm{~nm}$ ), while NIR spectroscopy uses input wavelengths from roughly $700 \mathrm{~nm}$ to $2500 \mathrm{~nm}$. Another characteristics of HSI is that 
it can generate a 3D image cube, where every pixel in a 2D image has also spectral values for all the wavelengths.

The physical stress test in the research requires the participant to do as many squats as possible within a certain period of time to trigger physical stress, and the participant will report their stress level after the test. For the data of 42 participants, the self-assessment stress levels are all greater than 6 points (rating 1 to 10 points), which means that 30 to 60 squats can indeed trigger physical stress for these participants. The maximal exercise is not achieved in this study, the physical stress induced in the research is not the stress induced by maximal exercise. In that case, the self-assessment levels could be as high as 10 .

The features used in the research are relatively simple and the classification task is two-class task (emotional stress or physical stress), we therefore choose SVM as the classifier because SVM is one of the simplest but classical classifiers suitable for two-class classification task. We also used other classical and commonly used machine learning algorithms, such as KNN and decision tree (see Table 4), for the classification. Compared to the SVM, they cannot achieve as high a classification accuracy as SVM. There will be of course other methods that can improve the classification accuracy. Researchers are encouraged to develop their own more sophisticated algorithms to reach higher classification rates on the published database.

To achieve high classification accuracy of stress detection, there are two factors. One is the algorithm used, the other is the input data. In this paper, what we would like to emphasize is that the input data ( $\mathrm{StO} 2)$ is a good signal for stress classification. To achieve this purpose, we have used a very simply algorithm to classify the two types of stress, i.e., calculating the average $\mathrm{StO} 2$ on ROIs as features, using SVM as the classifier. These algorithms are the simplest (or as simple as the others) in the Tables 5 and 6 . However, the algorithm can achieve the best classification accuracy. The results support our notion that facial $\mathrm{StO} 2$ can be used as a promising feature to identify stress states, including emotional and physical stress.

Since the importance of stress to human health cannot be ignored, it is essential to accurately detect stress and propose appropriate stress relief methods accordingly. In this paper, the three facial area features extracted by HSI can achieve an accuracy of more than $95 \%$ to distinguish between emotional stress and physical stress. This indicates that the facial $\mathrm{StO} 2$ feature is a valid physiological indicator of differentiating stress. The proposed method could be used to monitoring and discriminating human stress and suggest a proper treatment method according to the type of stress detected. It will also interesting to investigate in the future whether $\mathrm{StO} 2$ could be a potential feature for detecting basic emotions.

Author Contributions: Conceptualization, X.L. and T.C.; Methodology, X.L. and M.P.; Data analysis, X.L., M.P., H.C. and Y.S.; Validation, X.L. All authors wrote the paper. All authors have read and agreed to the published version of the manuscript.

Funding: This research received no external funding.

Acknowledgments: We would like to thank all the volunteers who join in the experiments as testees and all the graduated students in Affective Computing Group, Southwest University, who have spent lots of their time to collect the data.

Conflicts of Interest: The authors declare no conflict of interest.

\section{References}

1. Healey, J.; Picard, R.W. SmartCar: Detecting driver stress. In Proceedings of the International Conference on Pattern Recognition, Barcelona, Spain, 3-7 September 2000.

2. Kivimäki, M.; Virtanen, M.; Vartia, M.; Elovainio, M.; Vahtera, J.; Keltikangas-Jarvi, L. Workplace bullying and the risk of cardiovascular disease and depression. Occup. Environ. Med. 2003, 60, 779-783.

3. Schnall, P.; Belkić, K.; Landsbergis, P.; Baker, D. Why the workplace and cardiovascular disease? Occup. Med. 2000, 15, 1-5. [PubMed] 
4. Juster, R.; Mcewen, B.S.; Lupien, S.J. Allostatic load biomarkers of chronic stress and impact on health and cognition. Neurosci. Biobehav. Rev. 2010, 35, 2-16. [CrossRef]

5. Rugulies, R. Depression as a predictor for coronary heart disease: A review and meta-analysis. Am. J. Prev. Med. 2002, 23, 51-61. [CrossRef]

6. Anxiety and Physical Illness. Available online: https://www.health.harvard.edu/staying-healthy/anxiety_ and_physical_illness (accessed on 12 February 2016).

7. Thoits, P.A. Stress and health major findings and policy implications. J. Health Soc. Behav. 2010, 51. [CrossRef] [PubMed]

8. Widanti, N.; Sumanto, B.; Rosa, P.; Miftahudin, M.F. Stress level detection using heart rate, blood pressure, and GSR and stress therapy by utilizing infrared. In Proceedings of the International Conference on Industrial Instrumentation and Control, Pune, India, 28-30 May 2015.

9. Wen, W.; Liu, G.; Cheng, N.; Wei, J.; Shangguan, P.; Huang, W. Emotion recognition based on multi-variant correlation of physiological signals. IEEE Trans. Affect. Comput. 2014, 5, 126-140. [CrossRef]

10. Ren, P.; Barreto, A.; Gao, Y.; Adjouadi, M. Affective assessment by digital processing of the pupil diameter. IEEE Trans. Affect. Comput. 2012, 4, 2-14. [CrossRef]

11. Healey, J.; Picard, R.W. Detecting stress during real-world driving tasks using physiological sensors. IEEE Trans. Intell. Transp. Syst. 2005, 6, 156-166. [CrossRef]

12. Shastri, D.; Papadakis, M.; Tsiamyrtzis, P.; Bass, B.; Pavlidis, I. Perinasal imaging of physiological stress and its affective potential. IEEE Trans. Affect. Comput. 2012, 3, 366-378. [CrossRef]

13. Pavlidis, I.; Dowdall, J.; Sun, N.; Puri, C.; Fei, J.; Garbey, M. Interacting with Human physiology. Comput. Vis. Image Underst. 2007, 108, 150-170. [CrossRef]

14. Mcduff, D.; Gontarek, S.; Picard, R.W. Remote measurement of cognitive stress via heart rate variability. In Proceedings of the International Conference of the IEEE Engineering in Medicine and Biology Society, Chicago, IL, USA, 26-30 August 2014.

15. Chen, T.; Yuen, P.; Richardson, M.; Liu, G.; She, Z. Detection of psychological stress using a hyperspectral imaging technique. IEEE Trans. Affect. Comput. 2014, 5, 391-405. [CrossRef]

16. Hong, K.; Liu, X.; Liu, G.; Chen, W. Detection of physical stress using multispectral imaging. Neurocomputing 2019, 329, 116-128. [CrossRef]

17. Lundberg, U.; Forsman, M.; Zachau, G.; Eklöf, M.; Palmerud, G.; Melin, B.; Kadefors, R. Effects of experimentally induced mental and physical stress on motor unit recruitment in the trapezius muscle. Work Stress 2002, 16, 166-178. [CrossRef]

18. Coping with Stress. Centers for Disease Control and Prevention. Available online: https://www.cdc.gov/ violenceprevention/suicide/copingwith-stresstips.html (accessed on 12 February 2016).

19. Shan, Y.; Chen, T.; Yao, L.; Wu, Z.; Wen, W.; Liu, G. Remote detection and Classification of human stress using a depth sensing technique. In Proceedings of the Affective Computing and Intelligent Interaction, Beijing, China, 20-22 May 2018.

20. Shan, Y.; Li, S.; Chen, T. Respiratory signal and human stress: Non-contact detection of stress with a low-cost depth sensing camera. Int. J. Mach. Learn. Cybern. 2020. [CrossRef]

21. Liu, X.; Xiao, X.; Cao, R.; Chen, T. Evolution of facial tissue oxygen saturation and detection of human physical stress. In Proceedings of the Asia-Pacific Conference on Image Processing, Electronics and Computers (IPEC), Dalian, China, 14-16 April 2020.

22. Yuen, P.W.; Richardson, M.A. An introduction to hyperspectral imaging and its application for security, surveillance and target acquisition. Imaging Sci. J. 2010, 58, 241-253. [CrossRef]

23. Newman, J.D. MEMS programmable spectral imaging for remote sensing. SPIE Newsroom 2006, 4. [CrossRef]

24. Chen, T.; Yuen, P.; Richardson, M.; She, Z.; Liu, G. Wavelength and model selection for hyperspectral imaging of tissue oxygen saturation. Imaging Sci. J. 2015, 63, 290-295. [CrossRef]

25. Yuen, P.W.; Bishop, G.J. Hyperspectral multiple approach fusion for the long-range detection of low observable objects MUF2. In Proceedings of the International Society for Optics and Photonics, Stockholm, Sweden, 11-12 September 2006.

26. Chen, T.; Yuen, P.; Hong, K.; Ibrahim, I.; Tsitiridis, A.; Soori, U.; Jackman, J.; James, D.; Richardson, M. Assessment of tissue blood perfusion in-vitro using hyperspectral and thermal imaging techniques. In Proceedings of the International Conference on Bioinformatics and Biomedical Engineering, Wuhan, China, 10-12 May 2011. 
27. Wolf, M.; Von Siebenthal, K.; Keel, M.; Dietz, V.; Baenziger, O.; Bucher, H.U. Tissue oxygen saturation measured by near infrared spectrophotometry correlates with arterial oxygen saturation during induced oxygenation changes in neonates. Physiol. Meas. 2000, 21, 481-491. [CrossRef]

28. Rasmussen, P.; Dawson, E.; Nybo, L.; Van Lieshout, J.J.; Secher, N.H.; Gjedde, A. Capillary-oxygenationlevel-dependent near-infrared spectrometry in frontal lobe of humans. Br. J. Pharmacol. 2006, 27, 1082-1093. [CrossRef]

29. Yudovsky, D.; Nouvong, A.; Schomacker, K.; Pilon, L. Assessing diabetic foot ulcer development risk with hyperspectral tissue oximetry. J. Biomed. Opt. 2011, 16, 26009. [CrossRef]

30. Creates, M.S.; Grayson, J. The effect of adrenaline and noradrenaline on coronary vascular reserve in the dog. J. Physiol. 1980, 309, 557-568. [CrossRef]

31. Chen, T. Hyperspectral Imaging for the Remote Sensing of Blood Oxygenation and Emotions. Ph.D. Thesis, Cranfield University, Cranfield, UK, 2012.

32. Kirschbaum, C.; Pirke, K.M.; Hellhammer, D.H. The 'Trier Social Stress Test'-A tool for investigating psychobiological stress responses in a laboratory setting. Neuropsychobiology 1993, 28, 76-81. [CrossRef]

33. Golden, C.J.; Freshwater, S.M. Stroop Color and Word Test: A Manual for Clinical and Experimental Uses; Stoelting: Chicago, IL, USA, 1978.

34. Hjemdahl, P.; Freyschuss, U.; Juhlin-Dannfelt, A.; Linde, B. Differentiated sympathetic activation during mental stress evoked by the stroop test. Acta Physiol. Scand. 1984, 527, 25-29.

35. Tulen, J.H.M.; Moleman, P.; Van Steenis, H.G.; Boomsma, F. Characterization of stress reaction to the Stroop Color Word Test. Pharmacol. Biochem. Behav. 1989, 32, 9-15. [CrossRef]

36. Pavlidis, I.; Levine, J. Thermal image analysis for polygraph testing. IEEE Eng. Med. Boil. Mag. 2003, 21, 56-64. [CrossRef] [PubMed]

37. Van Der Zwaard, S.; Jaspers, R.T.; Blokland, I.J.; Achterberg, C.; Visser, J.M.; Uil, A.R.D.; Hofmijster, M.J.; Levels, K.; Noordhof, D.A.; De Haan, A.; et al. Oxygenation threshold derived from near-infrared spectroscopy: Reliability and its relationship with the first ventilatory threshold. PLoS ONE 2016, 11, e0162914. [CrossRef] [PubMed]

38. Chang, C.C.; Lin, C.J. LIBSVM: A Library for support vector machines. ACM Trans. Intell. Syst. Technol. 2011, 2,1-27. [CrossRef]

39. Benitez-Quiroz, C.F.; Srinivasan, R.; Martinez, A.M. Facial color is an efficient mechanism to visually transmit emotion. Proc. Natl. Acad. Sci. USA 2018, 115, 3581-3586. [CrossRef] [PubMed]

40. Sperry, R.W. Cerebral organization and behavior. Science 1961, 133, 1749-1757. [CrossRef] [PubMed]

41. Sperry, R.W. Hemisphere deconnection and unity in conscious awareness. Am. Psychol. 1968, 28, 723-733. [CrossRef]

42. Sperry, R.W. The Neurosciences: A Study Program; Rockefeller University Press: New York, NY, USA, 1967; pp. 714-722.

43. Lienhard, D.A. Roger Sperry's Split Brain Experiments; Arizona State University: Tempe, AZ, USA, 2017.

44. Zangroniz, R.; Martinez-Rodrigo, A.; Pastor, J.M.; Bonal, M.T.L.; Fernández-Caballero, A. Electrodermal activity sensor for classification of calm/distress condition. Sensors 2017, 17, 2324. [CrossRef] [PubMed]

45. Sriramprakash, S.; Prasanna, V.D.; Murthy, O.R. Stress detection in working people. Procedia Comput. Sci. 2017, 115, 359-366. [CrossRef]

46. Jebelli, H.; Hwang, S.; Lee, S. EEG-based workers' stress recognition at construction sites. Autom. Constr. 2018, 93, 315-324. [CrossRef]

47. Hong, K.; Liu, G.; Chen, W.; Hong, S. Classification of the emotional stress and physical stress using signal magnification and canonical correlation analysis. Pattern Recognit. 2018, 77, 140-149. [CrossRef]

48. Fernandez, J.R.M.; Anishchenko, L. Mental stress detection using bioradar respiratory signals. Biomed. Signal Process. Control 2018, 43, 244-249. [CrossRef]

49. Van Veen, R.L.P.; Sterenborg, H.J.C.M.; Pifferi, A.; Torricelli, A.; Chikoidze, E.; Cubeddu, R. Determination of visible near-IR absorption coefficients of mammalian fat using time- and spatially resolved diffuse reflectance and transmission spectroscopy. J. Biomed. Opt. 2005, 10, 054004. [CrossRef]

(C) 2020 by the authors. Licensee MDPI, Basel, Switzerland. This article is an open access article distributed under the terms and conditions of the Creative Commons Attribution (CC BY) license (http://creativecommons.org/licenses/by/4.0/). 